\title{
Growing Points in Leprosy Research
}

\section{(3) Immunological Detection of Sub-clinical Infection in Leprosy}

\author{
TORE GODAL \\ Immunologv Unit, World Health Organization, \\ Geneva, Switzerland
}

\begin{abstract}
The evidence for the presence of sub-clinical infection in leprosy, based on information acquired by the lymphocyte transformation test (LTT) and the leucocyte migration inhibition test (LMIT), is reviewed. These methods appear to have sufficient specificity to be useful as monitors of immune responses elicited by Mycobacterium leprae. The results obtained suggest that sub-clinical infection commonly follows exposure to Myco. leprae and therefore indicate that leprosy is more highly infectious than denoted by prevalence and incidence rates. Some observations imply that the intensity of exposure may modify host responsiveness to Myco. leprae.
\end{abstract}

In most, if not all, infectious diseases, apparently only a proportion of those who become exposed to the germ will develop the disease, while the rest will combat the infectious agent by developing effective immunity before it has time, either directly or indirectly, to cause overt disease. Such individuals are said to pass through the stage of sub-clinical infection. In some infectious diseases, e.g. poliomyelitis and tuberculosis, sub-clinical infection is a common outcome after exposure, while in others, such as smallpox, sub-clinical infection is a very rare event.

The epidemiological understanding of an infectious disease is dependent, to a very large extent, on how precisely the relationship between the infectious agent and each individual of a given population can be determined. At any one time subjects may be classified into either of the following categories:

(A) Not exposed.

(B) Exposed

(1) Non-immune: These individuals are incubating the germ but have not yet mounted an effective immune response.

(2) Immune: Such individuals may or may not harbour the germ. Their characteristic feature is that they have recovered and mounted an effective immune response subsequent to: (a) sub-clinical infection (no history of disease), or (b) clinical infection.

(3) Clinical disease.

The methods available for classification of a subject into either of the above-mentioned categories vary from one disease to the other. In general they are of three kinds:

(A) Detection of the infectious agent. This can be done quite accurately in 
diseases where the germ is harboured in an organ easily accessible for microbiological examination, such as the upper respiratory tract in the case of diphtheria. However, of ten the target organ is inaccessible to direct examination (e.g. lungs in tuberculosis) and the proportion of carriers, therefore, cannot be precisely determined.

(B) Detection of immune response. In diphtheria the presence of an effective (or protective) immune response may be assessed by toxin neutralizing skin reactivity (Schick test). However, in many other diseases, where the immune response to the infectious agent may be monitored, the response may be associated with, but not directly related to, protective immunity. This apparently applies to the tuberculin test in tuberculosis.

(C) When the above methods were lacking or unsatisfactory, attempts have been made to acquire information about the frequency of sub-clinical infection by searching for minor pathological changes in the target organ, e.g. slight alterations in liver function tests indicating sub-clinical hepatitis.

It should be noted that the estimation of the total proportion of a population which has been exposed to an infectious agent is usually dependent on all three categories of tests. Thus, only a proportion of exposed individuals can be detected by any one kind of test alone.

In leprosy also these three diferent types of approach have been applied.

(A) Detection of leprosy bacilli. Following the observations of Figueredo and Desai, (1949), Taylor, Elliston and Gideon (1965) searched for acid fast bacilli in the ear lobes of contacts of leprosy patients. They found $7 / 71$ (10\%) of the contacts of lepromatous patients and $2 / 80(2.5 \%)$ of the contacts of tuberculoid patients to be positive for acid fast bacilli, while out of 50 contacts examined in a leprosy non-endemic area none were found to be positive. Although direct evidence that these bacilli were in fact leprosy bacilli still appears to be lacking, "positive" contacts have subsequently been found to have a sixfold increased risk of acquiring leprosy (Chatterjee et al., 1973). This suggests not only that the acid fast bacilli were leprosy bacilli but also that many, if not most, of these "carriers" were in the incubation period of the disease.

(B) Detection of immune response to Myco. leprae. Previously the most widely used immunological test for examination of the immune response to Myco. leprae was the late (Mitsuda) lepromin test. However, because the test has been found to be positive in individuals not exposed to leprosy (see Rees, 1964) it has been disregarded as a useful test for detection of immune responses elicited by $M y c o$. leprae. Moreover, even with the early (Fernandez) reaction, which is read after 48-72 h, positive reactions have been observed in subjects from non-endemic areas (see Rees, 1964, Shepard and Saitz, 1967, Waters, 1973), although strong reactions appear to be much less common among non-contacts, including TB patients, than leprosy contacts (Rotberg, Bechelli and Keil, 1950). With the Fernandez reaction Waters found that only 1 out of 65 tuberculin negative volunteers reacted positively, while approximately $1 / 3$ of the tuberculin positive subjects showed positive reactions. These results indicate that there is a considerable degree of cross-reactivity between Myco. tuberculosis and autoclaved Myco. leprae. However, Myrvang (1973), who has done lepromin testing on a small number of contacts and non-contacts in Addis Ababa, found non-contacts uniformly negative with the Fernandez reaction, even though many responded strongly to BCG in vitro. In contrast, a high proportion (50\%) of occupational contacts responded to lepromin by the Fernandez reaction. 
(C) Detection of sub-clinical pathological changes in leprosy. It has been claimed that a large proportion of contacts have an enlargement of the larger auricular nerve (Karat, personal communication).

From these studies it appears difficult to draw any definite conclusions either because the results are conflicting, as is the case with the lepromin test, or because their relationship to leprosy remains unclear. Thus, there would appear to be a need for new methods to be found for the study of the epidemiology of leprosy.

Recently, methods by which it has become feasible to measure immune responses associated with cell-mediated immunity to Myco. leprae have been established in vitro. They include the lymphocyte transformation test (LTT) and the leucocyte migration inhibition test (LMIT). I shall now turn to discuss in some detail these tests and observations made in contacts of leprosy patients in order to try to reveal their potential as epidemiological tools in leprosy.

\section{Detection of Cell-mediated Immunity to Myco. leprae in vitro}

It is now a well-established fact that lymphocytes carry antigen receptors on their surface. When exposed to an antigen which can interact with the receptors, the lymphocyte, being either a $\mathrm{T}$ or a $\mathrm{B}$ cell, will enlarge and start to divide. This phenomenon is called blastoid transformation or simply lymphocyte transformation. Lymphocyte transformation may be monitored in vitro by counting the number of transformed cells morphologically or measuring the associated DNA synthesis by uptake of radioactive thymidine. If the stimulated cell is a B cell the end result will be antibody synthesis and generation of many $B$ cells with the same specificity. If the stimulated cell is a $\mathrm{T}$ cell, a more complex pattern and only partly understood chain of events will follow bringing about both cell-mediated immunity and a delayed-type hypersensitivity reaction in vivo. One of the early results of $\mathrm{T}$ cell stimulation is the liberation of molecular mediators (hereafter called lymphokines). The lymphokines have a number of key functions in the expression of cell-mediated immunity, one being their capacity to stimulate the macrophages to increased antibacterial activity (Godal, Rees and Lamvik, 1971 ; Fowles et al., 1973). The production and release of these lymphokines can be monitored in vitro by various tests, of which their ability to inhibit macrophages from migrating out of a capillary tube is the best established method. In man there is no easy access to macrophages, except for blood monocytes which are present in too small numbers for ordinary capillary tube methods. In search of methods applicable to man, the migration of buffy coat leucocytes (mainly granulocytes) has become utilized. However, the mechanism of this test and the involvement of lymphokines are not yet fully established. Moreover, it has been shown that humoral immune reactions can modify the test. Thus, it is evident that neither LTT nor LMIT is specific for delayed type hypersensitivity reactions. However, both tests have been found in man, in a number of test sytems, to correlate to delayed type hypersensitivity reaction. For more detailed information the reader is referred to recent reviews on the subject (Bloom, 1971; David and David, 1972; WHO, 1973).

The practical conclusions to be drawn from these observations are that neither the LMIT nor the LTT can a priori be judged as in vitro correlates to delayed type hypersensitivity and cell-mediated immune reactions. The existence of such a relationship has to be established in each individual test system.

Information regarding the relationship between LTT and LMIT delayed 
hypersensitivity and host resistance (cell-mediated immunity) is derived, in leprosy, mainly from studies of leprosy patients. It may, therefore, be pertinent to review briefly these studies before discussing the results obtained using these tests in contacts and non-contacts of leprosy patients.

\section{LTT and LMIT in Leprosy.Patients}

Immune responsiveness to Myco. leprae has been studied throughout the clinical and histopathological spectrum of leprosy both by LTT and LMIT (Myrvang et al., 1973). By both these methods, as well as the early and late lepromin reaction, the responses continuously decreased from the polar tuberculoid (TT) group to the polar lepromatous (LL) group. Thus, the overall picture revealed was that these tests are correlated to histological and clinical signs of host resistance. It is most likely that all these phenomena are related to the expression of $\mathrm{T}$ cell function to Myco. leprae antigens in these patients. On the contrary, B cell functions, as examined by the presence of precipitating antibody to other mycobacteria ( $M y c o$. leprae is not available in large enough quantities for the test), gradually increased from the TT to the LL end of the spectrum (Myrvang and Feek, 1973).

\section{LTT and LMIT in Healthy Contacts of Leprosy Patients}

\section{(a) MEDICAL ATTENDANTS}

Out of 122 medical attendants who had been working with leprosy patients, 71 ( $58 \%$ ) were found to respond to Myco. leprae by LTT $\geqslant 2 \%$.* Thirty-six of the 122 medical attendants tested were non-Ethiopian residents working at leprosy institutions in various parts of the world, who had come to ALERT to participate in courses organized there. Twenty out of the $36(56 \%)$ responded positively. Fifty-two individuals belonging to the medical attendant group were examined by LMIT. Thirty-seven $(71 \%)$ were found to give rise to $\leqslant 80 \%$ migration in the presence of Myco. leprae (Myrvang, 1973)

\section{(b) HOUSEHOLD CONTACTS}

Out of 105 subjects living in the same hut or compound as a leprosy patient, 49 (47\%) responded by LTT to Myco. leprae. The majority were family members of the patient and living in the same straw hut as the patient. Out of these 88 could be sub-divided, according to the diagnosis of the patient, into contacts of tuberculoid or indeterminate patients (29) and lepromatous (BL-LL) patients (59). Out of the 29 tuberculoid contacts, 18 (62\%) responded to Myco. leprae by LTT, while only $24 / 59(41 \%)$ of the lepromatous contacts gave a positive response (Godal and Negassi, 1973)! Further subdivision of the contacts of lepromatous patients provided suggestive evidence that contacts of treated patients responded better than contacts of untreated patients and that spouses behaved similarly to genetically related contacts. This was a most unexpected observation as obviously untreated lepromatous patients are the most infectious ones.

*It should be noted that this definition of a "responder" deviates from that of our recent publications (Godal, Löfgren and Negassi, 1972; Godal and Negassi, (1973) where the cross-reactivity to Myco. tuberculosis was also taken into account (this will be discussed below). 
Only 12 household contacts have been examined by LMIT (Myrvang, 1973). Six $(50 \%)$ gave a positive reaction. The restricted numbers investigated did not allow meaningful subdivision according to the diagnosis of the patient.

\section{LTT and LMIT in Non-contacts}

(a) SUBJECTS FROM NON-ENDEMIC AREAS

None of 26 individuals tested within 2 months of their arrival in Addis Ababa responded by LTT and all of 10 similar subjects were negative with LMIT.

\section{(b) SUBJECTS LIVING IN ENDEMIC AREAS WITHOUT ANAMNESTIC EVIDENCE OF CONTACT WITH LEPROSY PATIENTS}

Out of 45 subjects who had lived in Ethiopia for more than 1 year, 13 (29\%) responded by LTT, while none out of 7 of a similar group gave a positive response by LMIT to Myco. leprae (Myrvang, 1973). The LTT group was heterogeneous concerning duration of stay. Thirty-one were expatriates who had lived in Ethiopia between 1 and more than 10 years and 14 were Ethiopian medical personnel (nurses and dressers), most of them were working at the TB centre in Addis Ababa.

\section{On the Specificity of LTT and LMIT}

The most striking difference between the results obtained with LMIT and LTT and those obtained previously with the early lepromin reaction, was the uniform lack of response among definite non-contacts, i.e. individuals from non-endemic areas. This would indicate that LTT and LMIT are more specific than the early lepromin reaction. Thus, what is the evidence that LTT and LMIT are specific to Mycò. leprae vis-à-vis Myco. tuberculosis (var. humanus and BCG)?

(1) The average respone to Myco leprae among 12 nurses and dressers at the TB centre in Addis Ababa, who had been working with tuberculosis patients for more than 4 years, was only $3.08 \%$ as compared with a response of $21.1 \%$ to BCG. Since only two of them had been BCG vaccinated, this finding indicated that the tubercle bacillus only has a limited cross-reactivity with Myco. leprae in the LTT $(3.08 / 21.1=14.7 \%$ cross-reactivity) (Godal, Löfgren and Negassi, 1972). Since these individuals had been living in the endemic area all their lives, it was argued that these figures could show too high values as the subjects could have been exposed to Myco. leprae as well.

(2) A group of 8 subjects, who showed initially a low response to BCG (1.08\% mean) and to Myco. leprae (0.34\%), was vaccinated with BCG. This was followed by a rapid increase to a maximum of $9.16 \%$ one month after vaccination, while the response to $M y c o$. leprae remained low, showing a maximal response of $1.28 \%$ also one month after vaccination.

(3) In a study undertaken in Norway the response to Myco. leprae was studied in BCG vaccinated subjects (Closs, 1973). The responses to Myco. leprae were found to be related to the tuberculin reactivity of the subject and the mean cross-reactivity to Myco. leprae as compared to BCG was $27 \%$. However, the study is not strictly comparable to our studies in Addis Ababa as a micro-LTT method was used.

All these observations indicate that there is a high degree of specificity to 
Myco. leprae v. Myco. tuberculosis in the LTT. However, the studies of Closs, in particular seem to indicate that responses to Myco. tuberculosis may influence the responses to Myco. leprae and that the level of cross-reactivity may vary according to technique used. The influence of threshold level selected for cross-reactivity between Myco. tuberculosis and Myco. leprae on the proportion of responders in various groups is shown in Table 1 . By increasing the threshold of cross-reactivity from 0 to $50 \%$, the proportion of response to Myco. leprae falls from 29 to $2 \%$ while more than $30 \%$ of contacts remain responsive even at a $50 \%$ level. This is in spite of the fact that the non-contact group had a higher mean response to BCG than both the contact groups.

Another question is whether other mycobacteria in the environment in Addis Ababa include a mycobacterium with a much higher cross-reactivity to Myco. leprae. This seems unlikely for the following two reasons.

(1) The proportion of LTT responders among staff in Ethiopia (60\%) is virtually the same as the proportion of responders among occupational contacts from outside Ethiopia (56\%).

(2) A striking difference in responders to Myco. leprae between Ethiopian staff at ALERT has been found between those who are working with patients as compared to administration staff (Godal and Negassi, 1973).

The LMIT has only been studied in a more limited number of subjects. However, there appears to be no correlation in any group between responses to Myco. leprae and BCG. Thus, the LMIT might turn out to have even a higher degree of specificity than LTT (Myrvang, 1973).

These findings suggest that both LTT and LMIT appear to have sufficient specificity to become promising tools in epidemiological studies of leprosy. However, the specificity of the tests needs careful attention in each case. It might perhaps be improved by using sub-optimal concentrations of Myco. leprae and more purified antigenic preparations, as there is evidence that a more purified antigen increases the specificity of the Fernandez reaction (Dharmendra, 1948).

Although our observations at present do not allow any detailed analysis between the in vitro tests and the Fernandez reaction, the results suggest a higher specificity in the in vitro tests. One factor which may explain this difference is that fresh Myco. leprae is used in LTT and LMIT. Thus, it is possible that denaturation by autoclavation may make the antigens of $M y c o$. leprae less specific in the Fernandez reaction.

\section{Epidemiological Implications}

The results obtained with LTT and LMIT have provided a considerable amount of new evidence that sub-clinical infection occurs frequently in leprosy; in fact it appears to be the most common outcome after exposure. This concept is not new as a similar conclusion has been reached by the common observation that only a few of those who live together with a high infectious patient will acquire the disease (see Skinsnes, 1964). Our observations suggest that such individuals are not resistant, but develop resistance subsequent to infection.

It appears that leprosy is more contageous than indicated by the prevalence and incidence rates. However, the degree of infectiousness cannot be precisely determined for two reasons. Firstly, as outlined above, immunological methods will only detect a proportion of the exposed part of any population. Secondly, the interpretation of our results will depend on threshold level for cross-reactivity 


\section{TABLE 1}

The influence of various threshold levels of cross-reactivity between Myco. leprae and $B C G$ on per cent responders by lymphocyte transformation among contacts and non-contacts of leprosy patients

\begin{tabular}{lcccccc}
\hline $\begin{array}{l}\text { \% responders to Myco. leprae } \\
(\geqslant 2 \%)\end{array}$ & $\begin{array}{c}\text { \% responders to BCG } \\
(\geqslant 2 \%)\end{array}$ & $\begin{array}{c}\text { Mean responses to BCG } \\
(\% \text { transformation) }\end{array}$ \\
$\begin{array}{l}\text { Threshold level of cross-reactivity } \\
\text { Myco. leprae/BCG } \times 100)\end{array}$ & $0 \%$ & $15 \%$ & $30 \%$ & $50 \%$ & & 3.59 \\
\hline $\begin{array}{c}\text { Non-contacts from } \\
\text { non-endemic areas }\end{array}$ & 0 & 0 & 0 & 0 & 53 & 13.80 \\
$\begin{array}{c}\text { Non-contacts living in } \\
\text { endemic areas } \geqslant 1 \text { year }\end{array}$ & 29 & 24 & 9 & 2 & 78 & 11.57 \\
$\begin{array}{l}\text { Occupational contacts } \\
\text { Household contacts }\end{array}$ & 58 & 53 & 38 & 31 & 84 & 8.40 \\
\hline
\end{tabular}


to Myco. tuberculosis. If $15 \%$ is chosen, our data would indicate that a considerable proportion of individuals would become exposed without their knowledge when living in endemic areas. While if a threshold of $50 \%$ is chosen, only very few non-contacts living in an endemic area remain as responders. However, a considerable proportion of contacts would still be classified as "exposed".

In fact, since the LTT appeared in our hands to be quite specific to Myco. leprae, the cross-reactivity being in the order of $15 \%$ or less, I would favour the interpretations based on a $15 \%$ cross-reactivity level. Further support for this high degree of infectivity in leprosy may be found in the observation that only a small proportion of individuals know their source of infection (Badger, 1964) and the observations that patients with active lepromatous leprosy shed as many bacilli from the upper respiratory tract as an open case of tuberculosis (Davey and Rees, 1973). These observations may sound very alarming to medical personnel concerned with leprosy. However, it must be stressed that these observations do not change the fact that in any case only a very small proportion of medical attendants develop the disease.

The high proportion of response found among contacts of tuberculoid patients may be interpreted in two ways:

(1) Tuberculoid patients are infectious;

(2) Both the tuberculoid patient and the contact may have been exposed to an unknown lepromatous patient in the community.

Although the second explanation would seem more likely than the first one, our observations do not permit distinction between these two possibilities.

The low proportion of response found among contacts of active lepromatous patients was unexpected and would appear paradoxical. However, there have been similar findings with lepromin which showed that children who had close contacts with lepromatous patients had negative lepromin tests more of ten than children who had not had such contact (see Dharmendra, 1948). These findings raise several questions concerning risk factors in leprosy, other than exposure alone, as contributing factors in the susceptibility to leprosy, namely:

(1) Role of genetic factors.

(2) Intensity and nature of exposure: (a) initial dose, (b) duration of exposure, (c) route.

(3) Nutritional status of host.

(4) Age.

Recent genetic studies on twins suggests that genetic factors, although possibly contributing, do not play a decisive role in leprosy (Chakravartti and Vogel, 1973). Since spouses of lepromatous patients also responded poorly, genetic factors alone would not appear to explain our observations (Godal and Negassi, 1973).

Since our observations may indicate that contacts tend to recover when the patient is put on treatment, their suppressed response may be related to intensity of exposure, perhaps in combination with other suppressive factors on the immune system such as malnutrition.

These possibilities are at present hypothetical and can only be rejected or supported by further studies, not only in man but perhaps in experimental animals where the conditions can be much more precisely regulated to give specific answers in this very complex field. 


\section{References}

Badger, L. F. (1964). Epidemiology. In Leprosy in Theory and Practice, 2nd Ed. (Eds R. G. Cochrane and T. F. Davey), p. 69. Bristol: John Wright \& Sons.

Bloom, B. R. (1971). In vitro approaches to the mechanism of cell-mediated immune reactions. Adv. Immunol. 13, 102.

Chakravartti, M. R. and Vogel, F. (1973). A twin study on leprosy. Topics in Human Genetics 1,1 .

Chatterjee, B. R., Thomas, J., Taylor, C. E. and Naidu, G. N. (1973). Epidemiological findings of a longitudinal study in a defined population in Jhalda, West Bengal. All India Leprosy Workers' Conference Silver Jubilee and Centenary of Hansen's Bacillus Discovery, Sevagram, India. Abstracts, p. 5.

Closs, O. (1973). The reaction to PPD, BCG and lepromin in a population in which leprosy is non-endemic. In Abstracts of Tenth International Le prosy Congress, Bergen, p. 60.

Davey, T. F. and Rees, R. J. W. (1973). The nasal discharge in leprosy. Tenth International Leprosy Congress, Bergen. Abstracts, p. 30.

David, J. R. and David, R. R. (1972). Cellular hypersensitivity and immunity. Prog. Allergy. 16, 300.

Dharmendra (1948). The Lepromin Test. London: Belra Medical Series, No. 1.

Figueredo, N. and Desai, S. D. (1949). Positive bacillary findings in the skin of contacts of leprosy patients. Ind. J. Med. Sci. 4, 253.

Fowles, R. E., Fajardo, I. M., Leibowitch, J. L. and David, J. R. (1973). The enhancement of macrophage bacteriostasis by products of activated lymphocytes. J. exp. Med. 138, 952.

Godal, T., Rees, R. J. W. and Lamvik, J. O. (1971). Lymphocyte mediated modification of blood-derived macrophage function in vitro: including inhibition of growth of intracellular mycobacteria. Clin. Exp. Immunol. 8, 625.

Godal, T., Löfgren, M. and Negassi, K. (1972). Immune response to M. leprae of healthy leprosy contacts. Int. J. Lepr. 40, 243.

Godal, T, and Negassi, K. (1973). Subclinical infection in leprosy. Brit. med. J. II, 557.

Myrvang, B. (1973). Manuscript in preparation.

Myrvang, B., Godal, T., Ridley, D. S., Froland, S. S. and Song, Y. K. (1973). Immune responsiveness to Mycobacterium leprae and other mycobacterial antigens throughout the clinical and histopathological spectrum of leprosy. Clin. Exp. Immunol. 14, 541.

Myrvang, B. and Feek, C. M. (1973). Manuscript in preparation.

Rees, R. J. W. (1964).. The significance of the lepromin reaction in man. Prog. Allergy 8, 224.

Rotberg, A., Bechelli, L. M. and Keil, H. (1950). The Mitsuda reaction in a nonleprous area. Int. J. Lepr. 18, 209.

Shepard, C. C. and Saitz, E. W. (1967). Lepromin and tuberculin reactivity in adults not exposed to leprosy. J. Immunol. 99, 637.

Skinsnes, O. K. (1964). II. The immunological spectrum of leprosy. In Leprosy in Theory and Practice, 2nd Ed. (Eds Cochrane R. G. and Davey T. F.), p. 156. Bristol: John Wright \& Sons.

Taylor, C. E., Elliston, E. P. and Gideon, H. (1965). Asymptomatic infections in leprosy. Int. J. Lepr. 33, 716.

Waters, M. F. R. (1973). Significance of the lepromin test in tuberculin-negative volunteers permanently resident in a leprosy-free area. In Abstracts of Tenth International Leprosy Congress, Bergen, p. 81.

WHO, Technical Report Series No. 519 (1973). Cell-mediated Immunity and Resistance to Infection. Geneva. 